\title{
Monitoramento do binômio tempo e temperatura nos processos de produção de alimentos em um restaurante universitário
}

-Vanessa Gesser Correa vanesagesser@gmail.com Pós-Graduação em Ciência de Alimentos, Universidade Estadual de Maringá, Maringá Paraná, Brasil

Francieli Queiroz

francieliqueiroz@gmail.com

Departamento de Nutrição, Universidade Federal da Fronteira Sul, Câmpus Realeza, Realeza, Paraná, Brasil

\section{Edinéia Bonin}

edineiabonin42@hotmail.com

Pós-Graduação em Ciência de Alimentos, Universidade Estadual de Maringá, Maringá, Paraná, Brasil

Elis Carolina Souza Fatel elis.fatel@uffs.edu.br

elis.fatel@uffs.edu.br Federal da Fronteira Sul, Câmpus Realeza, Realeza, Paraná, Brasil

\section{Gabriela Bucaneve Guedes} gaby guedes@yahoo.com.br Departamento de Tecnologia em Gastronomia, União Educacional de
Cascavel, Cascavel, Paraná, Brasil

\begin{abstract}
RESUMO
O objetivo deste estudo foi avaliar o binômio tempo e temperatura nas diversas etapas do processo produtivo e analisar os riscos de sobrevivência e multiplicação de microrganismos nos alimentos oferecidos. Foram mensuradas durante cinco dias não consecutivos as temperaturas referentes ao recebimento, armazenamento, cocção e reaquecimento, distribuição e da cadeia de resfriamento, em um restaurante universitário localizado no oeste do Paraná. Observou-se que no processo de recebimento houve adequação em todas as mensurações. No armazenamento houve adequação total para as carnes congeladas, pass through frio e demais alimentos acondicionados no freezer, já os refrigeradores e o pass through quente obtiveram $60 \%$ de adequação e a câmara de hortifrútis e ovos $0 \%$ de conformidade. Em relação a cocção e reaquecimento notou-se que $66,6 \%$ das preparações estavam em conformidade e que dentre as não conformes houve grande participação de alimentos de risco, como os cárneos. Na distribuição, as inadequações relacionaram-se, principalmente, ao prato principal e as guarnições (13,3\% e $26,6 \%$ de conformidade, respectivamente). Na cadeia de resfriamento observou-se que nenhum grupo de alimento apresentou o procedimento de forma correta. O controle do tempo e temperatura é fundamental para a qualidade do produto final, dessa forma, é aconselhável que medidas de controle do binômio sejam implantadas diariamente e em todos os processos.
\end{abstract}

PALAVRAS-CHAVE: serviços de alimentação, segurança alimentar, conservação de alimentos, alimentação coletiva. 


\section{INTRODUÇÃO}

As Unidades de Alimentação e Nutrição (UANs) podem ser definidas como um serviço organizado que compreende uma sequência e sucessão de atos destinados a fornecer refeições balanceadas dentro dos padrões dietéticos e higiênicos, de modo que se ajustem aos limites financeiros da instituição (ABREU et al., 2009). Tais instituições tem o objetivo de oferecer alimentos com as qualidades necessárias a fim de promover, manter ou mesmo recuperar a saúde individual e coletiva dos usuários que se beneficiam da alimentação servida (ANTUNES et al., 2006).

A busca e a importância dos serviços das UANs têm ganhado destaque com o desenvolvimento e crescimento das populações que vivem nas cidades. Segundo o IBGE (2010), o consumo de alimentos realizado fora do domicílio tem aumentado no Brasil. Na Pesquisa de Orçamentos Familiares (POF) desenvolvida em 2008-2009 identificou-se que 31\% dos gastos com alimentação foram destinados à alimentação fora do domicilio contra 24\% em 2002-2003 (IBGE, 2010). As alterações no estilo de vida da população são decorrentes das novas demandas geradas pelo modo de vida urbano.

Diante deste crescente aumento do setor de alimentação fora de casa e devido ao risco que as más condições de manipulação podem trazer a saúde dos comensais, verifica-se a necessidade da implantação de métodos capazes de controlar a qualidade das refeições em suas diversas dimensões (RICARDO et al., 2012; FRANTZ et al., 2008). Nesse sentido, as Doenças Transmitidas por Alimentos (DTAs), definidas como um episódio onde duas ou mais pessoas apresentam o quadro clínico semelhante após ingerir alimentos ou água do mesmo local de origem que estavam contaminados, têm sua origem em procedimentos incorretos que se desenvolvem por falhas múltiplas relacionadas ao momento da produção (RICARDO et al., 2012).

A contaminação pode ser proveniente da matéria-prima contaminada antes do preparo, pela falha na higienização, manipuladores infectados/contaminados em contato direto com os alimentos, equipamentos contaminados e contaminação cruzada (SILVA JUNIOR, 2005). Já em relação a sobrevivência e a multiplicação dos microrganismos destacam-se falhas como cocção e reaquecimento insuficiente e a conservação em tempo e temperatura inadequados (CHESCA et al., 2001; OLIVEIRA et al., 2003).

Nesse sentido, o cuidado com a temperatura ideal é um dos meios mais eficazes na administração do crescimento de microrganismos e aliando-a com a monitorização do tempo se tem um dos binômios mais pesquisados no que se refere ao controle, eliminação ou diminuição do número de agentes patogênicos durante a produção de alimentos (SILVA JUNIOR, 2005). Destaca-se que esta quando descontrolada, pode estar relacionada às enfermidades de origem alimentar, como as intoxicações e infecções alimentares, além de poder causar a deterioração do alimento. Assim, o objetivo deste trabalho foi avaliar o binômio tempo e temperatura nas diversas etapas do processo produtivo, e analisar os riscos de sobrevivência e multiplicação de microrganismos nos alimentos oferecidos. 


\section{MATERIAIS E MÉTODOS}

O trabalho foi desenvolvido em uma UAN, localizada na região oeste do Paraná do tipo institucional, que distribui cerca de 1000 refeições diárias para o público universitário, tendo sido permitido o estudo por meio da assinatura de uma declaração de ciência e concordância das instituições envolvidas.

A coleta do tempo e da temperatura da distribuição foi realizada por cinco dias não consecutivos ao início, meio e fim durante o almoço. Foram mensuradas todas as preparações quentes e frias servidas e a água do balcão térmico. Nos grupos de preparações que apresentaram duas ou mais opções de prato foi realizada a média das temperaturas.

Para o monitoramento das demais etapas do processo produtivo foram mensuradas as temperaturas referentes ao recebimento de matérias primas, ao processo de resfriamento, equipamentos de congelamento, resfriamento e armazenamento durante a produção, durante cinco dias. A coleta da temperatura do recebimento foi realizada nos alimentos cárneos congelados no momento da recepção. Para a avaliação do resfriamento mensurou-se a temperatura logo que os alimentos foram submetidos ao processo e duas horas após. Os equipamentos de congelamento, resfriamento e armazenamento durante a produção (pass throught) foram monitorados de acordo com o termômetro do próprio equipamento e pela temperatura dos alimentos acondicionados nos mesmos. Por fim, a mensuração da temperatura de cocção e/ou reaquecimento foi realizada ao término da preparação.

Para a mensuração da temperatura utilizou-se termômetro digital tipo "espeto" da marca Incoterm ${ }^{\circledR}$, com capacidade de variação de -45 a $230^{\circ} \mathrm{C}$. Para a análise dos alimentos a haste do termômetro foi introduzida até o centro geométrico do mesmo e a temperatura anotada foi aquela que perdurou por 5 segundos. Após cada mensuração o termômetro foi higienizado com água e sabão neutro e álcool $70 \%$.

Para o controle e tratamento dos dados todas as temperaturas coletadas foram anotadas em planilhas específicas desenvolvidas para cada tipo de análise e tabuladas no programa Microsoft Excel ${ }^{\circledR} 2007$.

\section{RESULTADOS E DISCUSSÃO}

De acordo com ABERC (2003), no recebimento, os produtos cárneos congelados devem apresentar temperatura entre $-18{ }^{\circ} \mathrm{C}$ a $-12{ }^{\circ} \mathrm{C}$, contudo de acordo com os critérios de uso, os alimentos deste grupo que se apresentam entre $0 \stackrel{\circ}{\circ}$ a $5{ }^{\circ} \mathrm{C}$ podem ser utilizados em um prazo de validade de apenas 10 dias.

Os alimentos armazenados nos refrigeradores compreendiam os produtos frios, embutidos, maionese, sobremesas, preparações com laticínios, produtos de panificação e confeitaria e demais alimentos preparados. Assim, para a conservação dos alimentos a recomendação é que sejam expostos a $4{ }^{\circ} \mathrm{C}$ considerando os gêneros que necessitam de uma menor temperatura (SÃO PAULO, 2014; ABERC 2003). Em relação a temperatura dos equipamentos utilizados para a refrigeração, notou-se que no refrigerador 1 houve $0 \%$ de adequação, no refrigerador $2,80 \%$ e no $3,100 \%$ de conformidade (Tabela 1 ). 
Tabela 1. Temperatura de equipamentos $\left({ }^{\circ} \mathrm{C}\right)$ e índice de adequação.

\begin{tabular}{|c|c|c|c|c|c|c|c|}
\hline Equipamentos & $10 \mathrm{dia}^{\mathrm{a}}$ & $2^{\circ} \mathrm{dia}^{\mathrm{a}}$ & $30 \mathrm{dia}^{\mathrm{a}}$ & $4^{\circ} \mathrm{dia}^{\mathrm{a}}$ & 5o dia $^{a}$ & Médiab & Adequação \\
\hline Refrigerador 1 & 5 & 5 & 5 & 5 & 5 & 5 & $0 \%$ \\
\hline Refrigerador 2 & 2,8 & 2 & 4,8 & 3,4 & 3,6 & 3,3 & $80 \%$ \\
\hline Refrigerador 3 & 1,3 & 0,2 & 0,1 & 3,1 & 2,6 & 1,4 & $100 \%$ \\
\hline Câmara de hortifrútis e ovos & 16 & 16 & 16 & 16 & 16 & 16 & $0 \%$ \\
\hline Câmara de congelamento de carnes & -15 & -16 & -15 & -18 & -17 & $-16,2$ & $100 \%$ \\
\hline Freezer & -9 & -9 & -9 & -9 & -9 & -9 & $100 \%$ \\
\hline Pass through quente & 64,3 & 54,8 & 64,6 & 54,6 & 58,6 & 59,4 & $60 \%$ \\
\hline Pass through frio & 10 & 7 & 8 & 10 & 10 & 9 & $100 \%$ \\
\hline
\end{tabular}

NOTA: a Temperaturas diárias dos equipamentos analisados; ${ }^{\mathbf{b}}$ Média das temperaturas dos equipamentos analisados

Na câmara destinada ao armazenamento de hortifrútis e ovos observa-se que a temperatura apresenta $0 \%$ de adequação, visto que este equipamento está programado para $16{ }^{\circ} \mathrm{C}$, quando recomenda-se que estes gêneros alimentícios estejam a uma temperatura de $4{ }^{\circ} \mathrm{C}$ a $10{ }^{\circ} \mathrm{C}$, respectivamente (ABERC, 2003; SÃO PAULO 2013). O mesmo resultado foi encontrado por Dal Ri et al. (2011), que verificaram $100 \%$ de inadequação neste equipamento.

No que se refere aos equipamentos destinados ao congelamento dos gêneros alimentícios, observou-se que a câmara de carnes teve temperatura média de $16,2{ }^{\circ} \mathrm{C}$ e o freezer de $-9{ }^{\circ} \mathrm{C}$, observando $100 \%$ de adequação em ambos. De acordo com a recomendação de tempo e temperatura para estas condições (SÃO PAULO, 2013) os alimentos da câmara de congelamento têm um prazo de validade de aproximadamente 30 dias e os demais de 20 dias.

A temperatura adequada para a conservação a quente dos alimentos submetidos a cocção deve ser superior a $60{ }^{\circ} \mathrm{C}$ (ANVISA, 2004). Assim, é possível verificar que a temperatura do pass through quente esteve adequada em $60 \%$ das mensurações. Em estudo realizado por Dal Ri et al. (2011), a adequação do mesmo alcançou $83,3 \%$ e $75 \%$ nos dois equipamentos analisados. Já Soares, Monteiro e Schaefer (2009), observaram 60\% de adequação. No que se refere as mensurações do pass through frio, observou-se $100 \%$ de adequação, tendo em vista que a recomendação para armazenamento desses alimentos é de até $10{ }^{\circ} \mathrm{C}$ por 4 horas e de $10^{\circ} \mathrm{C}$ a $21^{\circ} \mathrm{C}$ por 2 horas (ABERC, 2003; SÃO PAULO 2013).

Para a cocção e reaquecimento dos alimentos, verifica-se que todas as partes dos alimentos devem atingir a temperatura de $70{ }^{\circ} \mathrm{C}$ no centro geométrico (ANVISA, 2004). Nas mensurações realizadas pós-cocção e reaquecimento observou-se que de acordo com a análise das temperaturas de todos os alimentos submetidos a esses processos houve $66,6 \%$ de conformidade. Os alimentos com temperatura abaixo do ideal foram os pratos principais: coxa e sobrecoxa de frango com molho, filé de peixe frito, frango grelhado, carne suína acebolada e as guarnições suflê de legumes e farofa de ovo. Em relação a estes resultados destaca-se que os produtos com temperaturas inadequadas são em 
sua grande maioria produtos cárneos, que são frequentemente envolvidos na contaminação por microrganismos patogênicos devido aos mesmos serem ricos em nutrientes e conter elevada quantidade de água (BRASIL, 1997).

O tempo de distribuição dos alimentos foi de aproximadamente duas horas. Foram mensuradas as temperaturas das preparações quentes e frias servidas pelo estabelecimento e da água do balcão nos dias analisados e apresentadas suas médias com base no item e nos três horários de distribuição (Tabela 2). O percentual de adequação se refere a todas a temperaturas mensuradas para a realização das médias.

Tabela 2. Média do tempo e temperaturas $\left({ }^{\circ} \mathrm{C}\right)$ da distribuição.

\begin{tabular}{lcccc}
\hline Item & 10 Horário & 20 Horário & 30 Horário & \% de adequação \\
\hline Média do horário & $11: 15$ & $12: 30$ & $13: 34$ & - \\
Arroz branco & 69,5 & 66,0 & 63,1 & $66,6 \%$ \\
Feijão & 71,0 & 58,1 & 56,1 & $53,3 \%$ \\
Guarnição & 50,4 & 54,3 & 54,1 & $26,6 \%$ \\
Prato principal & 54,9 & 49,3 & 47,1 & $13,3 \%$ \\
Pratos frios & 17,4 & 19,2 & 20,2 & $73,3 \%$ \\
Água do balcão & 47,6 & 95,2 & 94,0 & $66,6 \%$ \\
\hline
\end{tabular}

Segundo a RDC 216, após serem submetidos à cocção, os alimentos devem ser mantidos a uma temperatura superior a $60{ }^{\circ} \mathrm{C}$ por no máximo 6 horas, respeitando as condições de tempo e de temperatura que não favoreçam a multiplicação microbiana (ANVISA, 2004).

Em relação à temperatura de distribuição dos alimentos quentes pode-se observar que o arroz branco manteve a média adequada nos três momentos de mensuração, contudo, o percentual de adequação considerando todas as mensurações, foi de 66,6\%. Em estudo realizado por Soares, Monteiro e Schaefer (2009) foi observado $100 \%$ de adequação para o arroz integral e $80 \%$ para o branco.

No que se refere ao feijão, verifica-se que a média das temperaturas iniciais apresentou-se acima dos $60{ }^{\circ} \mathrm{C}$, mas que no decorrer da distribuição houve a redução, chegando a $56,1{ }^{\circ} \mathrm{C}$. Foi possível observar que este alimento permaneceu, em alguns dos dias analisados, exposto em temperatura ambiente após a cocção. Assim, esta pode ser uma possível explicação para os resultados encontrados. No estudo desenvolvido por Soares et al., (2009), a temperatura de distribuição do feijão apresentou $100 \%$ de adequação, isto porque na unidade analisada foi verificada a eficiência do carrinho térmico utilizado para manter o calor e o fato de que esta preparação foi a última a ser finalizada.

A guarnição e prato principal não atingiram a temperatura desejável nos três momentos analisados, sendo que foram os itens alimentares que apresentam os menores percentuais de adequação considerando todas as 
mensurações realizadas $(26,6 \%$ e $13,3 \%$, respectivamente). A temperatura adequada, tanto no armazenamento como na distribuição, é um dos fatores que podem contribuir para a garantia da qualidade das refeições servidas, minimizando os riscos de contaminação e crescimento microbiológico (MONTEIRO et al., 2014). Trindade et al. (2008) verificaram em seu estudo que as carnes e as guarnições foram os gêneros alimentícios com maiores irregularidades, o mesmo foi observado por Ricardo et al. (2012), no qual os dois itens alimentares apresentaram maiores inadequações nas mensurações intermediárias e final. Em estudos realizados por Dal Ri et al. (2011), e Soares et al. (2009), a guarnição foi a preparação que apresentou o menor percentual de adequação atingindo $30,8 \%$ e $26,7 \%$, respectivamente.

Em estudo realizado por Monteiro et al. (2014), as preparações quentes obtiveram uma média de $56{ }^{\circ} \mathrm{C}$, os autores relacionaram os resultados encontrados a fatores como a ausência de controle da temperatura da espera para a distribuição, equipamentos inadequados de aquecimento e a falta de mecanismos de controle. No presente estudo uma possível explicação para que as temperaturas tenham apresentado valores abaixo do desejado está também relacionada a falhas no acondicionamento anterior a distribuição, sendo que os alimentos encontravam-se dispostos em cima dos fogões, dentro dos fornos e em outros lugares à temperatura ambiente. Ainda, a temperatura do pass through pode ter sido insuficiente para a conservação da mesma nos alimentos, conforme verificado no monitoramento dos equipamentos.

Em relação às preparações frias de acordo com a Portaria CVS 05/2013 pode-se alcançar a temperatura entre $10^{\circ} \mathrm{C}$ e $21^{\circ} \mathrm{C}$ por um período máximo de duas horas (SÃO PAULO, 2014). Assim, verifica-se que a média nas três mensurações esteve em conformidade e que ainda, este grupo apresentou melhor porcentagem de adequação $(73,3 \%)$ em relação aos demais. Destaca-se que nos dias analisados, a temperatura do ambiente estava baixa, o que pode ter contribuído para que estes estivessem nos valores desejados, sendo que no restaurante avaliado não há a exposição destes alimentos em balcão refrigerado e em alguns dias houve a disposição destes itens no balcão anexo ao quente.

No estudo realizado por Dal Ri et al. (2008), dos alimentos frios verificados, apenas $5 \%$ estavam em conformidade, sendo o mesmo analisado por Ventimiglia e Basso, (2008), os quais observaram inadequação da temperatura de saladas servidas em restaurantes.

A água do balcão térmico deve ser trocada diariamente e mantida a temperatura de $80{ }^{\circ} \mathrm{C}$ a $90{ }^{\circ} \mathrm{C}$ devendo ser mensurada durante o tempo de distribuição (SÃO PAULO, 2014). No que concerne a média das temperaturas mensuradas nos três momentos, verifica-se que houve inconformidade somente ao início da distribuição, resultando assim em $66,6 \%$ de adequação. Este fato pode ser explicado pelo intervalo de tempo insuficiente desde que 0 equipamento é ligado até a montagem do buffet, sendo em média 10 minutos. Soares et al. (2009), observaram em seu estudo a média da temperatura da água de $74,3{ }^{\circ} \mathrm{C}$, estando, portanto, em inconformidade. Na pesquisa desenvolvida por Dal Ri et al. (2011), houve um percentual de conformidade de $33 \%$ e $41,6 \%$ nos dois buffets analisados. O resultado foi ainda mais preocupante na análise desenvolvida por Ricardo et al. (2012), na qual foi observado $100 \%$ de inadequação. 
Para a correta realização da cadeia de resfriamento nota-se que a temperatura do alimento preparado deve ser reduzida de $60{ }^{\circ} \mathrm{C}$ a $10{ }^{\circ} \mathrm{C}$ em até duas horas, em seguida, o mesmo deve ser conservado sob refrigeração a temperaturas inferiores a $5^{\circ} \mathrm{C}$, ou congelado à temperatura igual ou inferior a $18^{\circ} \mathrm{C}$ (SÃO PAULO, 2014). No que se refere aos hortifrútis, a recomendação é de que os mesmos voltem a temperatura de $4^{\circ} \mathrm{C}$ (ABERC, 2003).

Conforme descrito na Figura 1, no que se refere a cadeia de resfriamento do arroz branco e o feijão verifica-se que a média da temperatura inicial esteve próxima do ideal, contudo a final permaneceu significativamente acima do recomendado. Em relação as carnes e as guarnições, observou-se que temperatura inicial e a final apresentaram-se distantes da recomendação.

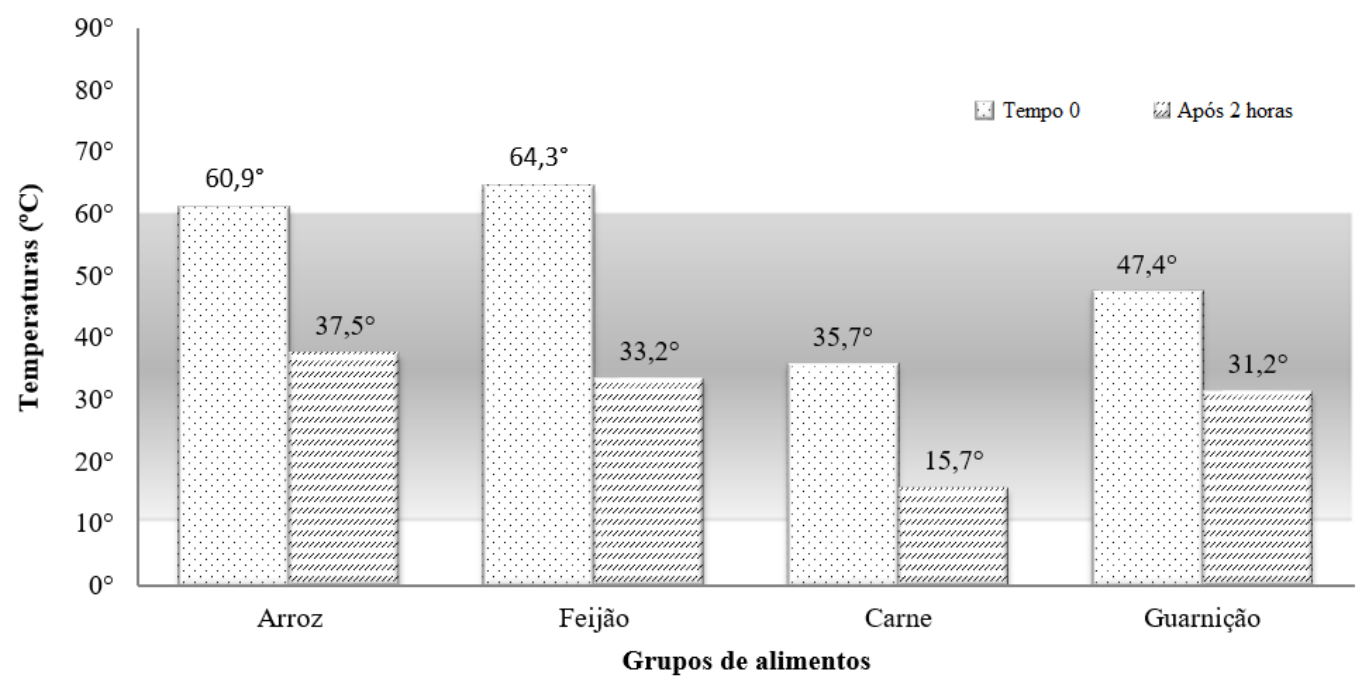

Figura 1. Média das temperaturas da cadeia de resfriamento. NOTA: O destaque na área de plotagem refere-se a faixa de temperatura ideal da cadeia de resfriamento $\left(60^{\circ} \mathrm{C}\right.$ a $\left.10^{\circ} \mathrm{C}\right)$.

Uma possível explicação para os resultados é que para o resfriamento, os alimentos são acondicionados em equipamentos com baixa capacidade para a redução da temperatura e assim, o processo ocorre de forma mais lenta do que o recomendado.

A realização da cadeia de resfriamento de forma inadequada traz prejuízos a qualidade do produto final e riscos à saúde do comensal. O processo de resfriamento de um alimento preparado deve ser realizado de forma a minimizar o risco de contaminação cruzada e a permanência do mesmo em temperaturas que favoreçam a multiplicação microbiana, inibindo o crescimento de microrganismos patogênicos e reduzindo a perda de água (SÃO PAULO, 2014).

\section{CONCLUSÃO}

De acordo com os resultados encontrados, um número significativo de processos envolvidos na produção das refeições apresentou inadequações de 
acordo com as legislações vigentes, que são agravadas por relacionarem-se a produtos com grande risco de contaminação, como os cárneos e hortifrútis.

Foi possível identificar que as inconformidades encontradas são devidas a falhas no processo após a cocção do alimento, com a regulagem da temperatura e manutenção dos equipamentos.

O controle do tempo e da temperatura é fundamental para a qualidade do produto final e para que se evite a proliferação de microrganismos patogênicos, garantindo a segurança do consumidor. Dessa forma é aconselhável que medidas de controle do binômio tempo e temperatura sejam implantadas diariamente e em todos os processos da produção de refeições. 


\title{
Monitoring of time and temperature binomial in food production process in an academic restaurant
}

\begin{abstract}
The aim of this study was to evaluate both time and temperature at different stages of the production process, and analyze the survival and multiplication risks of microorganisms in food. Temperatures on the receipt, storage, cooking and reheating, distribution and cooling chain were measured for five non-consecutive days in an academic restaurant located in western Parana. It was observed that the receiving process was adequate in all measurements. The storage was fully adequate for frozen meat, pass through cold and other packaged foods in the freezer, but, at the same time, refrigerators and hot pass through obtained only $60 \%$ of adequacy and, grocers chamber and eggs, obtained none conformity $(0 \%)$. Regarding to baking and reheating it was noted that $66.6 \%$ of the preparations were in conformity, and that among those nonconforming, there was great participation of risk foods such as meat. At distribution, the inadequacies were mainly related to the main course and the trimmings $(13.3 \%$ and $26.6 \%$ of conformity, respectively). In the cooling chain it was observed that no food group exhibited the procedure correctly. The control of time and temperature is crucial for the quality of the final product, thus, it is advised that measures control of both, time and temperature, must be implemented on a daily basis and in all processes.
\end{abstract}

KEYWORDS: Food Services, Food Security, Food Preservation, Collective Feeding. 


\section{REFERÊNCIAS}

BRASIL. Instrução Normativa $n^{\circ} 24$, de 10 de outubro de 2002. Instituto Brasileiro do Meio Ambiente e dos Recursos Naturais Renováveis- Ibama. Brasília: 2002a. Disponível em: < http://www.mma.gov.br/>. Acesso em: 12 jun. 2015.

BRASIL. Portaria $n^{\circ} 146$, de 7 de março de 1996. Regulamentos Técnicos de Identidade e Qualidade dos Produtos Lácteos. Brasília: 1996. Disponível em: <http://www.agricultura.gov.br/>. Acesso em: 11 jun. 2015.

BRASIL. Portaria no 27: Regulamentos técnicos de identidade e qualidade do queijo tropical. Brasília: 2002b. Disponível em: <http://www.agricultura.gov.br/>. Acesso em: 11 jun. 2015

FRISVAD, J. C.; SAMSON, R. A. Polyphasic taxonomy of Penicillium subgenus Penicillium. A guide to identification of food and air-borne terverticillate Penicillia and their mycotoxins. Studies in Mycology, v. 49, p. 1-173, 2004.

GERMANO, P. M. L., GERMANO, M. I. S. Higiene e vigilância sanitária de alimentos: qualidade das matérias-primas, doenças transmitidas por alimentos e treinamento de recursos humanos. 3ed., rev. e ampl. Barueri (SP): Manole, 2008.

GUSSO, A. P. Salga de queijos - uma revisão. In: I Encontro de Divulgação Científica e Tecnológica. Toledo, Paraná: Anais, p. 70-74, 2009.

HOCKING, A. D.; FAEDO, M. Fungi causing thread mould spoilage of vacuum packaged cheddar cheese during maturation. International Journal of Food Microbiology. v.16, p. 126-130, 1992.

KURE, C. F.; SKAAR, I.; BRENDEHAUG, J. Mould contamination in production of semi-hard cheese. International Journal of Food Microbiology. v. 93, p. 41-49, 2004.

LUND, F.; FILTENBORG, O.; FRISVAD, J. C. Associated mycoflora of cheese. Food Microbiology, v. 12, p. 173-180, 1995.

MAGALHÃES, F. A. R. Evolução de características físico-químicas e sensoriais durante a maturação do queijo tipo gorgonzola. 85 p. Tese (Doutorado) Universidade de Lavras, Lavras, 2002. 
MORAIS, V. M. F. Identificação de fungos leveduriformes e filamentosos em queijos de manteiga. 70 p. Dissertação (Mestrado) - Universidade Federal da Paraíba, João Pessoa, 2005.

NETO, J. S. C. Bolores deteriorantes em queijo parmesão. 65 p. Dissertação (Mestrado) - Universidade Estadual Paulista “Júlio de Mesquita Filho”, Araraquara, 2005.

PEIXOTO, J. P. N.; FURTADO, D. A.; OlIVEIRA, C. J. B.; GOMES, J. P. Qualidade do ambiente e níveis de contaminação por micro-organismos em queijarias, no Agreste Paraibano. Revista Brasileira de Produtos Agroindustriais, v.14, n. 2, p. 177-183, 2012.

PERRY, K. S. O. Queijos: aspectos químicos, bioquímicos e microbiológicos. Química Nova. v. 27, n. 2, p. 293-300, 2004.

PITT, J. I.; HOCKING, A. D. Fungi and Food Spoilage. London: Blackie Academic \& Professional, 2009.

TEMELLI, S.; ANAR, S.; SEN, C.; AKYUVA, P. Determination of microbiological contamination sources during Turkish white cheese production. Food Control, v. 17, p. 856-861, 2006.

TORKAR, K. G.; VENGUST, A. The presence of yeasts, moulds and aflatoxin M1 in raw milk and cheese in Slovenia. Food Control, v. 19, n. 5, p. 570-577, 2008.

ZACARCHENCO, P. B.; TRENTO, F. K. H.; SPADOTI, L. M.; GALLINA, D. A.; SILVA, A. T. Bolores e Leveduras em Queijos. TecnoLat-Expresso, Ano II, n. 8, p. 92-99, 2011.

Recebido: 26 out. 2016

Aprovado: 16 out. 2017

DOI: $10.3895 /$ rebrapa.v8n2.4886

Como citar:

CORREA, V. G. et al. Monitoramento do binômio tempo e temperatura nos processos de produção de

alimentos em um restaurante universitário. Brazilian Journal of Food Research, Campo Mourão, v. 8, n. 2,

p. 46-56, abr./jun. 2017. Disponível em: https://periodicos.utfpr.edu.br/rebrapa

Correspondência:

Vanessa Gesser Correa

Pós-Graduação em Ciência de Alimentos, Universidade Estadual de Maringá, Maringá, Paraná, Brasil

Direito autoral: Este artigo está licenciado sob os termos da Licença Creative Commons-Atribuição 4.0 Internacional.

(c) (i) 